\title{
Tech Transformation Strategy for Banks to Compete with Fintechs Sudipta Kumar Ghosh
}

Director of Software Engineering, Capital One, Chicago, Illinois, USA

\begin{abstract}
Article Info

Volume 8, Issue 5

Page Number : 47-51

Publication Issue :

September-October-2021

Article History

Accepted : 02 Sep 2021

Published: 07 Sep 2021

In the past decade, we saw tech innovation disrupting every industry. Banks, which maintained their dominant position because of complex regulations, are now seeing a new set of threats emerging in the form of Fintechs. To compete with Fintechs, banks need a Tech transformation strategy. That necessitates more than building a website or an app. It needs to incorporate digital technologies in every phase of the customer experience, move its infrastructure to the cloud, adopt Agile and DevOps practices, etc. This paper will discuss the key strategies that will help banks compete with Fintechs, including Cloud transformation, using digital technologies to create personalized customer experiences, facilitating faster product launch time, and attracting top talent.

Keywords : Tech Transformation, Cloud Transformation, Digital Transformation, Customer Experience, Cloud Computing
\end{abstract}

\section{INTRODUCTION}

The banking ecosystem that we have today is made up of many ancient processes and platforms. These processes are time-consuming, non-digital in nature, and provides poor customer experience. These processes are supported by aging enterprise-wide applications running on the bank's on-premises infrastructure, making it extremely difficult to add any innovative capabilities. On the other side, Fintehcs are app/mobile-based companies that run on flexible, scalable software on cloud infrastructures. They provide superior, and personal customer experiences, innovative products and are primarily digital in nature. Over the last decade, we saw Fintechs such as Venmo, Paypal, Quicken Loans, etc., rapidly gaining market share and become an enormous threat to the banking industry. To compete with the Fintechs, banks have no choice but to embark on a tech transformation journey.

\section{Overview of Fintechs}

In the past decade, we saw tremendous advancement in digital technologies. This disrupted the ways businesses and individuals perform financial activities. 
A new wave of technology-enabled firms offering financial services used this opportunity to develop alternative platforms for financial activities. These firms are called Fintechs, and they operate in various traditional banking spaces, including mobile payments, digital currencies, peer-to-peer lending, marketplace lending, etc. FinTechs usually focus on mobile functionality, big data, accessibility, agility, cloud computing, contextuality, personalization, and convenience. Traditional banks have been slow to react to the fintech revolution due to their heavy dependency on monolithic platforms and legacy infrastructure.

\section{Tech Strategy for Banks}

With Fintechs disrupting the banking industry, banks are left with no choice but to innovate. The latest cornerstone survey shows that $45 \%$ of banks have not launched a digital transformation strategy yet. Implementing such a huge transformation strategy is indeed easier said than done. The are several reasons for a company executive to feel not confident to make this investment. One of the key reasons is that many executives do not understand where to start - is it cloud transformation or digital transformation, or agile transformation? This paper will attempt to clarify the steps required to undergo tech transformation and help banks compete with Fintechs.

When did your institution launch its digital transformation strategy?

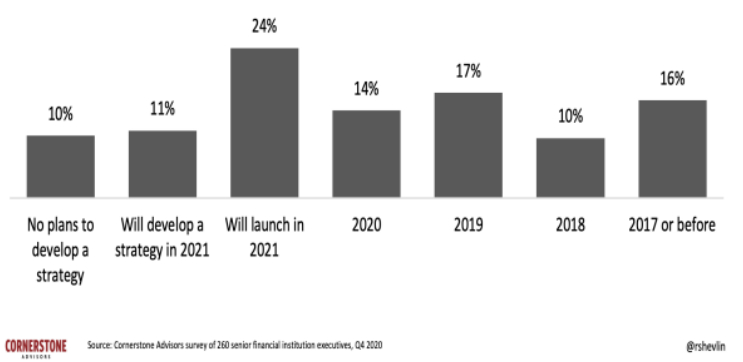

Figure 1. When did your institution launch its digital transformation strategy?

\section{Build a World-Class Tech-Force}

Most transformations fail because banks make a mistake to start a tech transformation without having the right skilled talent in the organization. A recent survey by GlobalData shows lack of specific skills and talents is the top reason for digital transformation failure.

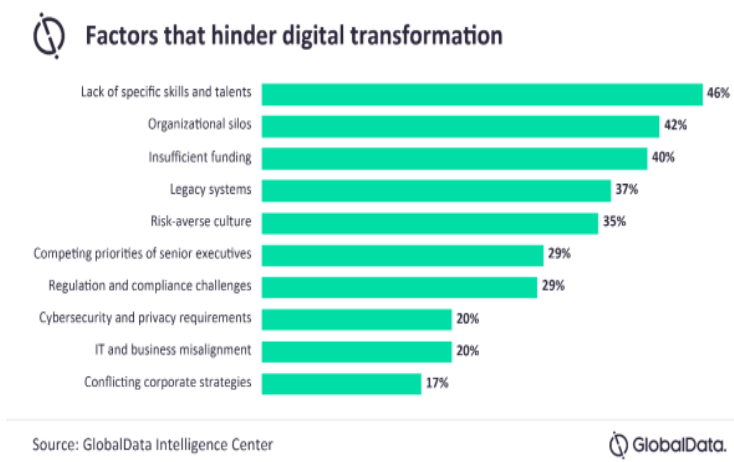

Figure 2. Factors that hinder digital transformation

Banks need top-class talent to embark on any transformation journey. People are the real heroes that make transformation happen, so a bank should invest heavily in recruiting and retaining top talents. The traditional bank's Technology Organizational model had several gaps. Talents were part of teams that have limited and narrow responsibilities. This model was not collaborative and slowed things down as one team waited for another to complete a request. Also, most banks have their IT outsourced to vendors. The challenge with this model is that it fails to build a culture of innovation and continuous learning within the organization. The vendor's resources also never had any appreciation for the Company's strategic needs.

To compete with the Fintachs, a Bank needs to change its talent model fundamentally. Banks need to recruit skilled engineers and provide existing talent with quality training, helping them skill up. They will also need to provide growth tracks for the talent, including non-managerial career tracks where 
engineers can continue to work on technology and earn manager-level salaries.

With top talent now in-house, banks need to organize them into small cross-functional teams to solve diverse problems and build innovative solutions. The teams are empowered to make decisions, manage endto-end delivery of capabilities and share their learnings with other teams to improve organization efficiency.

\section{Use Agile Software Development Principles :}

Once the bank has the good talent to embark on a tech transformation journey, now is the time to define operating principles. Traditionally bank's software development process was based on the waterfall model, which is a linear model. In this model, a phase has to be completed before moving on to the next stage. This model has several flaws. Poor risk management is one of the biggest and costliest concerns because defects are identified late in the process, which becomes more expensive to fix. Also, due to the nature of big bang releases, users don't get to provide feedback early on, resulting in missing features or incorrect functionalities being deployed to production. Adding or changing requirements is also a big challenge in this model.

Modern tech companies follow the Agile methodology of software development. This method focuses on an iterative and incremental way of developing software. Technology teams develop incremental versions of new products, get feedback from stakeholders, make improvements that users want, and continue this process until the product is finally delivered. This iterative and incremental process ensures that organizations deliver value by building what the users want and reducing costs by identifying defects early on.

\section{Move to Cloud:}

It's hard to believe that many banks are still hesitant when it comes to cloud transformation. Most leaders think of cloud transformation as an infrastructure decision; however, it's much more. Cloud transformation enables personalized, real-time customer experiences, game-changing data, and analytics, which increase customer satisfaction and loyalty. Let's look at the top 3 benefits of migrating to the cloud:

a) Operational Efficiency: Moving away from the on-premise data center to cloud infrastructure increases operational efficiency and decreases tech infrastructure cost. The infrastructure is maintained and upgraded by the cloud service provider helping companies to focus on building innovative capabilities that matter most. This also reduces non-labor costs by decreasing or eliminating datacenter related spending. Oneclick provisioning of infrastructure fast tracks capability launch time.

b) Scalability: One of the most significant benefits of the cloud is unlimited scale, both in terms of storage and compute power. As banks compete with Fintechs, they need to launch new innovative products regularly. With unlimited storage and compute capability, infrastructure will never be a bottleneck again.

c) Innovation: Migrating to the cloud opens up a bank's ability to use various innovative technologies on demand that helps create gamechanging customer experiences.

Now that we know the value cloud drives let's look at the top 3 strategies that a bank should implement as part of cloud transformation.

a) Identify Cloud Service Provider(s) : Banks can choose one or multiple Cloud Service Providers for capabilities to be hosted on the cloud. AWS, Google Cloud, Microsoft Azure are the key players in this market. A key consideration a bank should consider while choosing a provider is that the cybersecurity requirements are aligned with the bank's overall security strategy. 
b) Identify capabilities to be migrated to the cloud: Not all capabilities need to be moved to the cloud, nor all should move at once. The first wave of capabilities should ideally be customer-facing applications or heavy analytical tasks. These use cases need heavy storage or computing powers that need to scale up or down on demand, hence truly realizing the benefits of the cloud.

c) Define governance: Setting up a governance model is key to success. The governance model will define what access controls engineers should have, what technology/cloud capabilities are approved for usage, alerting and monitoring on infrastructure and costs, training developers on controls, how infrastructure is provisioned, etc. Without a robust governance model, banks will struggle to maintain a uniform operating standard across the organization.

\section{Provide superior user experience with Digital Transformation}

Financial institutions that are still on legacy architecture/platforms deprive customers of superior digital experiences. Most banks think of legacy modernization as infrastructure investment, but few know how moving to digital/cloud can help banks build game-changing customer experiences. It requires a paradigm shift that recognizes the value of giving customers the ability to choose a personalized experience in every interaction. Let's look at some of the strategies banks can implement to move become a digital organization :

a) Rethink the Customer Journey: One mistake most banks make is doing a lift-shift of their applications to the cloud and building a web UI / Mobile App and thinking they have achieved tech transformation. However, if we put a customer lens on this, there is no change in customer experience. The experience is still time-consuming, partly digital, and confusing.
Now compare that with the customer experience of Venmo, one of the largest Fintech that does Peer-Peer money transfers. The experience is entirely frictionless, search a user and Pay the user. It's a two-click experience. Banks need to redesign their platforms to provide such a frictionless digital experience.

b) Leverage data and analytics: One of the biggest advantages of digital transformation is collecting customer interaction/transaction data and running analytics on it. This helps the bank understand their customer behavior, opportunities to cross-sell new products to the customer, identify gaps in consumer experience, plug them with innovative products, and provide a more personable experience to each customer.

c) Build a digital organization: Bank should not stop at platform redesign but try to redesign their whole tech organization. This is not a once-anddone effort but a continuous investment to create a culture of innovation and continuous learning. Digital organizations build microservices that are linked with easy-toconfigure APIs and are hosted in the cloud. This provides the ability to replace the code if upgrades need to be done quickly. Digital organizations use DevOps principles with heavily automated deployment and testing processes. They focus on faster development time, high reusability, control on infrastructure provisions, and the use of CICD.

\section{CONCLUSION}

Though there are materials around each of the steps, a holistic framework to achieve tech transformation was missing, this paper has tried to address that. Following the above strategy will help a bank get started on the tech transformation journey. 


\section{REFERENCES}

[1]. GlobalData, "Digital Transformation and Emerging Technology in the Healthcare Industry - 2020 Edition"

[2]. Andrei Charniauski. and Lawrence Freeborn., "Cloud Banking - Innovation without limits."

[3]. Ron Shevlin ., "WHAT'S GOING ON IN BANKING 2020"

\section{Cite this article as :}

Sudipta Kumar Ghosh, "Tech Transformation Strategy for Banks to Compete with Fintechs", International Journal of Scientific Research in Science, Engineering and Technology (IJSRSET), Online ISSN : 2394-4099, Print ISSN : 2395-1990, Volume 8 Issue 5, pp. 47-51, September-October 2021. Available at doi : https://doi.org/10.32628/IJSRSET218485

Journal URL : https://ijsrset.com/IJSRSET218485 